\title{
Effekte der Kindererziehung auf Erwerbsprofile und Alterseinkommen von Frauen in West- und Ostdeutschland
}

Kindererziehung und Familienarbeit bergen für Frauen die Gefahr, dass im Alter ein finanziell auskömmliches Leben häufig nur durch den Ehepartner gesichert werden kann. Besonders Frauen in Westdeutschland mit mehreren Kindern sind davon betroffen. Empirische Analysen auf Basis von Daten der Rentenversicherung zeigen, dass die Kindererziehung deutliche Spuren in den Erwerbsbiografien von Müttern hinterlässt, die letztlich auch die Höhe der Renten beeinflussen. Die Versorgungslücken können durch besondere Leistungen der gesetzlichen Rentenversicherung nur bedingt ausgeglichen werden.

\section{Einleitung}

Verfolgt man die aktuelle sozialpolitische Debatte, dann schwebt das Damoklesschwert der Überalterung der Gesellschaft über der Zukunft der sozialen Sicherungssysteme. Im Windschatten dieser grundsätzlichen Diskussion erfahren auch die Forderung und Förderung der Vereinbarkeit von Kindererziehung und Erwerbstätigkeit neuen Aufwind. Für die Zukunft wird die Notwendigkeit gesehen, das Arbeitskräftepotenzial, das die nicht erwerbstätigen Frauen bilden, möglichst auszuschöpfen. Die Vereinbarkeit von Beruf und Familie wird dabei aus Sicht der Systeme der Alterssicherung als volkswirtschaftliches Ziel postuliert, um die Relation von Beitragszahlern zu Leistungsempfängern von Alterssicherungen günstiger zu gestalten (Stegmann 2004).

Dies trifft zusammen mit einem anderen Impetus, der sich aus der Veränderung der postulierten Rollenbilder von Mann und Frau ergibt. Das Aufbrechen der Orientierung der sozialen Sicherung am Leitbild der traditionellen Arbeitsteilung führte in der sozialpolitischen Debatte bereits seit mehr als drei Dekaden zur Forderung, die Erwerbstätigkeit von Frauen und Müttern zu stärken. Im Licht der gesellschaftlich gewandelten Sicht wird eine eigenständige, vom Partner unabhängige soziale Absicherung von Frauen gefordert. In diesem Kontext wird dann - über egalitäre Partizipationsmöglichkeiten am Erwerbsleben hinaus - eine stärkere Anerkennung der Familienarbeit in den Systemen der so- zialen Sicherung und besonders auch in der gesetzlichen Rentenversicherung postuliert (Stegmann 2001).

In der rentenpolitischen Diskussion werden häufig die Kindererziehung und die damit verbundene Unterbrechung, Reduzierung oder gar endgültige Beendigung der Erwerbstätigkeit als wesentliches Merkmal für deutlich geringere Rentenanwartschaften genannt (Pabel 1998). Im Zentrum steht damit die Frage nach dem $\mathrm{Zu}-$ sammenhang zwischen Kindererziehung und Erwerbsbeteiligung und den Auswirkungen dieser Faktoren auf die Altersvorsorge von Frauen. Die bis dato beste Analysebasis zur Untersuchung dieser Zusammenhänge bietet die Studie „Altersvorsorge in Deutschland (AViD) 1996" (Kortmann/Schatz 1999), die auf Längsschnittdaten der gesetzlichen Rentenversicherung aufbaut und die Geburtsjahrgänge 1936 bis 1955 erfasst. Die Ergebnisse sind repräsentativ für den Anteil der Bevölkerung, der eine Rente aus der Gesetzlichen Rentenversicherung (GRV) erhält. In Ostdeutschland sind dies $99 \%$ der Bevölkerung, in Westdeutschland etwa $90 \%$, weil hier bei Beamten und einigen besonderen Berufsgruppen wie Ärzten, Rechtsanwälten und anderen Stelbstständigen sich das Alterseinkommen hauptsächlich aus anderen Quellen speist.

Der Beitrag stellt zunächst dar, in welcher Form Erwerbstätigkeit und Kindererziehung in der gesetzlichen Rentenversicherung registriert werden und welche Analysemöglichkeiten die für die Untersuchung verwendeten Daten damit bieten (Rahn/Becker 1997; Ruland 2001; Krauthausen 2002). Danach werden Analysen mit den Längsschnittdaten für Frauen der
Jahrgänge 1936 bis 1955 zu deren Erwerbsverlauf und zum projizierten Alterseinkommen durchgeführt.

\section{Erwerbstätigkeit und Kindererziehung in den Daten der GRV}

Die gesetzliche Rentenversicherung berechnet die Leistungen der Altersrenten im Wesentlichen auf der Grundlage von Beiträgen und Beitragszeiten (Polster 1998). Diese werden vor allem durch die Höhe des Einkommens und die Dauer der Erwerbstätigkeit determiniert. Hinzu kommen vollwertige Beiträge für besondere Umstände, vor allem für Kindererziehung oder Pflege. Diese rentenrechtliche Bedeutung bringt es mit sich, dass die gesetzliche Rentenversicherung exakte Daten über die Erwerbstätigkeitsepisoden einer Person in ihrer Versicherungsbiografie hat. Bekannt sind jeweils Beginn und Ende sowie die Einkommenshöhe. Aus demselben Grund sind auch andere soziale Situationen exakt nachvollziehbar, unter anderem die Kindererziehung und die Anzahl der erzoge-

\footnotetext{
Michael Stegmann, Dr., Referent im Forschungsdatenzentrum der Rentenversicherung (FDZ-RV), Würzburg. Arbeitsschwerpunkte: Soziale Sicherung von Frauen und Familien, empirische Sozialforschung, Armutsforschung und soziale Sicherheit.

e-mail: michael.stegmann@drv-bund.de
} 
nen Kinder. In den Längsschnittdatensätzen, die auch der Studie „Altersvorsorge in Deutschland“ zugrunde liegen, ist die gesamte Versicherungsbiografie abgebildet. Mit diesen Datensätzen können daher Ereignisse, wie die Geburt eines Kindes, in ihrem biografischen Kontext, z. B. vorausgehende Erwerbstätigkeit oder Ausbildung und nachfolgende Arbeitslosigkeit oder Erwerbstätigkeit, betrachtet werden.

\subsection{VERSICHERUNGSPFLICHTIGE ERWERBSTÄTIGKEIT}

Die gesetzliche Rente orientiert sich in Deutschland vor allem an der Höhe der Beiträge und der Dauer der versicherungspflichtigen Erwerbstätigkeit: Wer viel einzahlt, soll auch verhältnismäßig mehr Rente bekommen als der, der vergleichsweise wenig einzahlt. Dies bezeichnet man als Teilhabeäquivalenz. Versicherungspflichtig war für die betrachteten Jahrgänge jede abhängige Beschäftigung, deren Bezahlung die Geringfügigkeit überschreitet. Darüber hinaus sind einige freie Berufe versicherungspflichtig, beispielsweise Hebammen oder Lehrende an Privatschulen. Keine Beiträge leisten Beamtinnen und Beamte, weil die staatliche Pension bei ihnen an die Stelle einer gesetzlichen Rente tritt, ferner z. B. Ärztinnen und Ärzte sowie Rechtsanwältinnen und Rechtsanwälte als Angehörige von Berufen, die einem berufsständischen Versorgungswerk für ihren speziellen Berufsstand angehören. Zum Kreis der versicherungspflichtigen Selbstständigen gehören dagegen beispielsweise HandwerkerInnen (Ausstieg frühestens nach 18 Beitragsjahren), die sich damit auch in den Daten der gesetzlichen Rentenversicherung finden.

Für jeden Monat einer versicherungspflichtigen Beschäftigung wird ermittelt, wie hoch das Einkommen im Verhältnis zum Durchschnitt aller sozialversicherungspflichtigen Einkommen in der Bevölkerung ist. ${ }^{1}$ Erzielt eine Person ein Jahr lang ein durchschnittliches Einkommen, dann wird ihr ein Entgeltpunkt auf ihrem Rentenversicherungskonto gutgeschrieben. Die Daten der Rentenversicherung zeigen damit die Höhe des Einkommens der Vergangenheit im Verhältnis zum Durchschnittseinkommen des jeweiligen Bezugsjahres. Die Entgeltpunkte ermöglichen eine sehr einfache Interpretation der relativen Einkommensposition. Allerdings bilden die Entgeltpunkte das Einkommen nur bis zu der Grenze ab, bis zu der Beiträge an die gesetzliche Rentenversicherung $\mathrm{zu}$ entrichten sind: die Beitragsbemessungsgrenze. Damit sind Analysen der Spitzeneinkommensbezieher mit den Daten nicht möglich. Weil Frauen nur sehr selten Einkommen in Höhe der Beitragsbemessungsgrenze beziehen, ist dies für die nachfolgende Analyse nur von geringer Bedeutung.

Für die Höhe der Altersrente spielt es keine Rolle, ob die Entgeltpunkte durch Erwerbstätigkeit mit stark schwankendem oder gleichmäßigem Einkommen erzielt wurden. Grundsätzlich ist es auch nicht wichtig, ob die Einzahlungen in einem langen, eher unterdurchschnittlich bezahlten oder in einem kurzen, hoch bezahlten Erwerbsleben entrichtet wurden. Die Summe der für eine Person ermittelten Entgeltpunkte ergibt mit dem jeweils aktuellen Rentenwert, ${ }^{2}$ dem Zugangsfaktor und dem Rentenartfaktor multipliziert die ausgezahlte Rente.

\subsection{KINDERERZIEHUNGSZEITEN}

Das sozialpolitische Ziel der Maßnahme, Zeiten der Kindererziehung in die Rentenberechnung aufzunehmen, war es, den Einkommensausfall, der durch Kindererziehung entsteht, teilweise zu kompensieren. Die Rentenversicherung erkennt für Frauen aller Geburtsjahrgänge ab 1921 in Westdeutschland und ab 1927 in Ostdeutschland die Erziehung eigener Kinder und außerdem die Erziehung von Adoptivund Pflegekindern an. Für jedes Kind, das vor 1992 geboren wurde, erhält die Erziehungsperson (in der Regel die Mutter) für ein Jahr die Rentenanwartschaften eines Durchschnittsverdieners. Für Kinder, die nach 1992 geboren wurden, beträgt dieser Zeitraum drei Jahre. Dies gilt grundsätzlich auch, wenn zusätzlich einer Erwerbstätigkeit nachgegangen wird. Die Kindererziehungsleistung kann allerdings immer nur einem Elternteil zugeordnet werden. Diesen Bonus erhält jede Erziehungsperson, wenn sie nicht in einem anderen Regelalterssicherungssystem abgesichert ist (z. B. Beamte). Weil der Nachweis von Kindererziehungszeiten die Rente steigert, kann von einer fast vollständigen Meldung der Geburten an den Rentenversicherungsträger ausgegangen werden.

\section{Datenbasis der Analysen}

Die Erhebung Altersvorsorge in Deutschland (AVID) 1996 zielte auf eine Verbesserung der empirischen Informationslage der GRV zu einer Reihe von Tatbeständen, vor allem zum Aufbau der Altersvorsorge. Die dargestellten Ergebnisse zu längsschnittbezogenen Biografiedaten basieren auf einer Stichprobe und für die Zeiten nach dem Befragungsjahr 1996 bis zum jeweils 65. Lebensjahr auf einer statistischen Fortschreibung. ${ }^{3}$ Das Design der Studie und die methodische Umsetzung werden ausführlich im Methodenband zur Untersuchung beschrieben (Kortmann et al. 2000). Einige wesentliche Rahmenbedingungen und methodische Besonderheiten des behandelten Themas werden im Folgenden kurz zusammengefasst.

Der Untersuchung AVID 1996 liegen eine repräsentative Befragung und Informationen aus dem Rentenversicherungskonto zugrunde. Aus den zusammengeführten Daten erfolgte eine Fortschreibung der Lebens- und Erwerbsverläufe bis zum jeweiligen 65. Lebensjahr auf Basis eines von Infratest Sozialforschung entwickelten Mikrosimulationsmodells. Der abschließende Schritt bestand in der Berechnung der Art und Höhe von Anwartschaften auf Versichertenrenten der GRV und Leistungen aller wichtigen Alterssicherungssysteme. Die Grundgesamtheit besteht aus den

Das Durchschnittseinkommen wird vom Statistischen Bundesamt errechnet und der Rentenversicherung mitgeteilt, die es ihren Berechnungen zugrunde legt.

2 Der aktuelle Rentenwert ist der Betrag, der einem Entgeltpunkt in der gesetzlichen Rentenversicherung entspricht. Seit dem 01.07.2003 beträgt er bislang unverändert $26,13 €$ für Rentenanwartschaften, die in den alten Bundesländern erworben wurden. Er wird jeweils zum 01.07. eines Jahres im Rahmen der Rentenanpassung neu bestimmt. Bei Zeiten, für die aufgrund einer Tätigkeit in den neuen Bundesländern oder der ehemaligen DDR Entgeltpunkte (Ost) ermittelt wurden, tritt anstelle des aktuellen Rentenwertes der aktuelle Rentenwert (Ost). Dieser beträgt seit 01.07.2003 $22,97 €$. Der aktuelle Rentenwert (Ost) steht zu dem aktuellen Rentenwert in dem Verhältnis, in dem die Nettolöhne und Gehälter in den neuen Bundesländern zu denen in den alten Bundesländern stehen.

3 Dies gilt in der Regel dann, wenn über die Höhe der Anwartschaften bzw. den gesamten Biografieverlauf berichtet wird. 
in Deutschland wohnenden deutschen Versicherten der GRV der Geburtsjahrgänge 1936-1955 und deren Ehepartnern, Letztere unabhängig von Nationalität, Alter oder Zugehörigkeit zur GRV. ${ }^{4}$ Sie schließt in den neuen Bundesländern nahezu die gesamte Bevölkerung dieser Jahrgänge ein. In den alten Bundesländern entfallen etwa $12 \%$ der Bevölkerung auf nicht durch die Untersuchung repräsentierte Personen ohne GRV-Konto sowie auf AusländerInnen ohne deutschen Ehepartner (Kortmann/ Schatz 1999, S. 34). Personen ohne Beteiligung an der GRV und damit auch ohne jeglichen Rentenanspruch sind in den folgenden Analysen nicht vertreten.

Das Fortschreibungsmodell der AVID simuliert die Erwerbs- und Nichterwerbsverläufe von Männern und Frauen bis zum 65. Lebensjahr. Einbezogen werden Ereignisse, die außerhalb der Entscheidungsfreiheit der GRV-Versicherten liegen, wie z. B. Altersarbeitslosigkeit und länger andauernde Krankheiten nach Auslaufen der Lohnfortzahlung. Ebenfalls berücksichtigt werden individuelle Entscheidungen zur Unterbrechung oder Beendigung der Erwerbstätigkeit, etwa zur Haushaltsführung oder Pflege eines Angehörigen, die nicht zu einem gleichzeitigen Bezug von Alterseinkommen führen. Übergänge in den Ruhestand vor oder nach Vollendung des 65 . Lebensjahres aus eigener Entscheidung und die damit verbundenen Abschläge wurden nicht simuliert. Rentenzugänge vor dem 65. Lebensjahr finden lediglich im Fall von Erwerbsunfähigkeit statt (Kortmann/ Schatz 1999, S. 57).

\section{Erwerbstätigkeitsprofile von kindererziehenden und kinderlosen Frauen}

Von einem Profil lässt sich dann sprechen, wenn wesentliche Charakteristika zu einem prägenden und abgegrenzten Gesamtbild verbunden werden. Es geht also nicht darum, Konturen und Schattierungen verschiedener oder typisierender Biografiemuster herauszuarbeiten. Vor dem Hintergrund der Fragestellung werden der $\mathrm{Zu}-$ sammenhang von Phasen der Kindererziehung bzw. der Erwerbsbeteiligung und die Auswirkungen auf das Alterseinkommen in den Vordergrund gestellt. Betrachtet werden Quoten und Durchschnitte für

Abb. 1: Lebensaltersbezogene Erwerbsquoten der Frauen in Westdeutschland (nach Anzahl der Kinder) - Geburtsjahrgänge 1936-1955 mit eigener projizierter GRV-Rente -

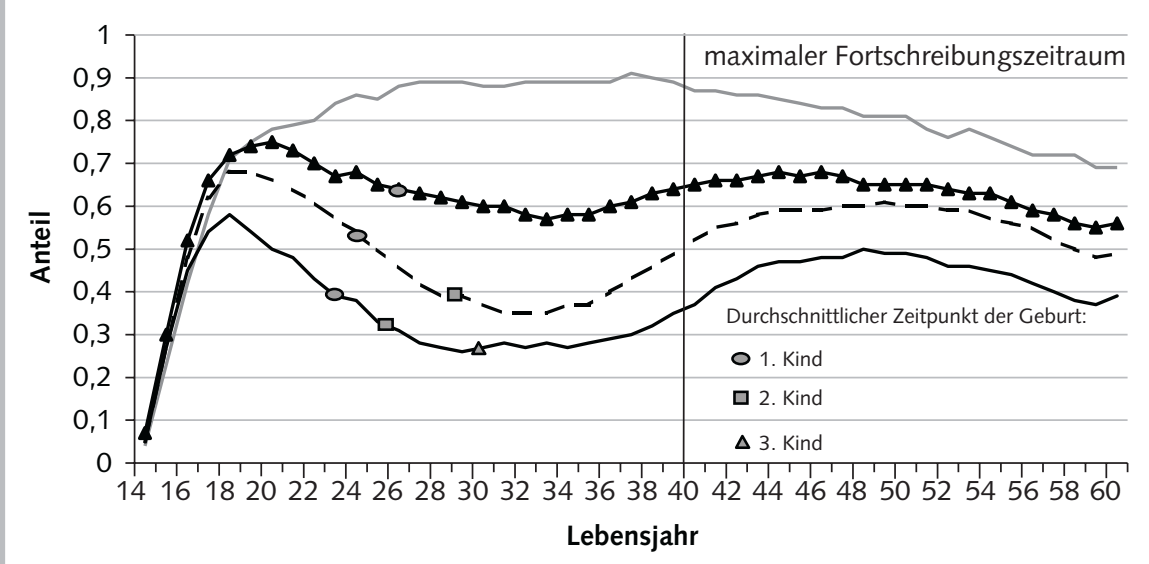

keine Kinder $\longrightarrow$ ein Kind $-ー-$ zwei Kinder $\longrightarrow$ drei Kinder u.m.

Anm.: Maximaler Fortschreibungszeitraum beschreibt den maximalen Zeitraum, der durch die

Mikrosimulation mit statistisch ermittelten Werten gefüllt ist. Dieser ist am längsten beim Jahrgang

1955.

Quelle: Altersvorsorge in Deutschland 1996 (AVID 1996); Berechnungen des Autors.

WSI Hans Böckler

Frauen mit dem prägenden Charakteristikum der unterschiedlichen Anzahl an Kindern. Auf eine Unterscheidung nach Geburtskohorten wird verzichtet, da sich keine signifikanten Differenzen ergeben.

\subsection{ERWERBSBETEILIGUNG}

Der Einfluss der Kindererziehung auf die Erwerbsbeteiligung zeigt sich in den unterschiedlichen Erwerbsbeteiligungen von kinderlosen Frauen und Frauen mit Kindern. Zunächst wird die altersbezogene Erwerbsquote analysiert.

In Westdeutschland sind deutliche Unterschiede zwischen kinderlosen Frauen und Müttern vorhanden. Die im Längsschnitt betrachtete altersbezogene Erwerbsquote $^{5}$ der kinderlosen Frauen steigt dort kontinuierlich bis zum 26. Lebensjahr an und erreicht im Maximum $90 \%$. Bis ca. zum 40. Lebensjahr wird dieses Niveau gehalten, um anschließend kontinuierlich abzusinken. Bei den Frauen mit Kindern zeigt sich dagegen früh ein kinderbezogener Rückgang der Erwerbsquote, der in Abhängigkeit von der Anzahl der Kinder gravierender und nachhaltiger ausfällt (Abbildung 1). Ein Bezug zur Kindererziehung lässt sich dabei in der Grafik durch das abgebildete durchschnittliche Alter bei Geburt der Kinder herstellen.

In Westdeutschland ist allen Frauen mit Kindern gemeinsam, dass nach einem anfänglichen Anstieg der Erwerbsquoten be- reits ab bzw. kurz nach dem 20. Lebensjahr die Erwerbsquoten wieder absinken, was jeweils vor dem durchschnittlichen Zeitpunkt der Geburt des ersten Kindes liegt. Dabei ist festzustellen, dass das erste Maximum der Erwerbsquoten umso niedriger ausfällt, je mehr Kinder die Frauen haben. Durch Kindererziehung bedingt, kommt es bei den Frauen mit einem Kind zu einem Absinken der altersbezogenen Erwerbsquote auf ein Minimum von ca. $58 \%$, das im 33. Lebensjahr erreicht wird. In den folgenden Lebensjahren steigt die Erwerbsquote wieder an und erreicht nochmals fast $70 \%$. Den wesentlich prägnanteren Unterschied zu den Frauen ohne Kinder weisen jedoch die Frauen mit zwei Kindern und die Frauen mit drei und mehr Kindern auf. Infolge der Kindererziehung kommt es bei ihnen zu einem regelrechten Einbruch der

4 Zu den Versicherten der GRV zählen hier auch jene, für die zwar aufgrund vergangener Versicherungszeiten ein Rentenversicherungskonto eingerichtet wurde, die aber, z. B. weil sie zum Erhebungszeitpunkt Hausfrauen oder Beamte sind, keine aktive Versicherungszeit aufweisen, sogenannte passiv Versicherte (VDR 1998).

5 Die Erwerbsquoten sind auf die Erwerbstätigkeit im jeweiligen Alter bezogen. Zu Erwerbsbeteiligten werden Personen gezählt, die mindestens sechs Monate im entsprechenden Kalenderjahr erwerbstätig waren. Die Erwerbsquote stellt das Verhältnis der Erwerbstätigen (sozialversicherungspflichtig Beschäftigte, Beamte, Selbstständige) zur gesamten Population dar. 

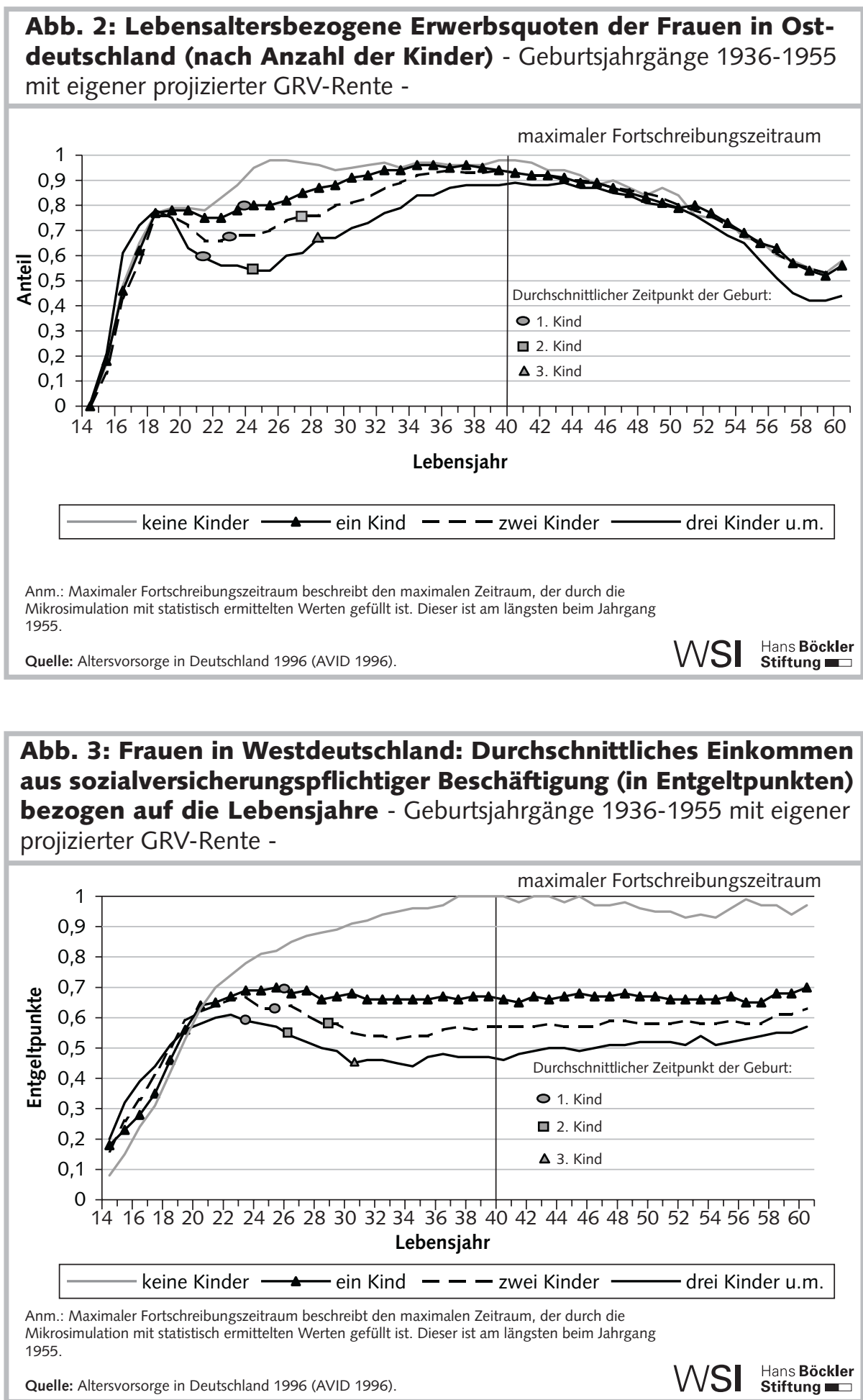

Erwerbsquote. Im 33. Lebensjahr sind von den Frauen mit zwei Kindern nur noch $35 \%$ erwerbstätig. Bei den Frauen mit drei und mehr Kindern liegt das Minimum der berufstätigen Frauen bereits im 30. Lebensjahr und erreicht nur noch $28 \%$. Ab Mitte bis Ende 30 beginnen die Erwerbsquoten wieder anzusteigen, erreichen aber bei den Frauen mit zwei Kindern nur noch maximal $60 \%$ und bei den Frauen mit drei und mehr Kindern $50 \%$. deckungsgleicher Verlauf der Erwerbsquoten; knapp $80 \%$ der Frauen sind in diesem Alter erwerbstätig. Danach differieren die Erwerbsquoten in Abhängigkeit von der Anzahl der Kinder. Die Erwerbsquote der kinderlosen Frauen steigt weiter an bis auf nahezu $100 \%$. Auf diesem Niveau, das im 22. Lebensjahr erreicht wird, verharrt die Erwerbsquote bis Mitte 40. Ab diesem Alter sinkt die Quote stetig und deutlich ab. Dieser letzte Befund gilt unabhängig von der Anzahl der Kinder und steht in deutlichem Zusammenhang mit dem Geschehen auf dem Arbeitsmarkt nach der Wende (Hauschild 2002).

Bei den Frauen mit Kindern kommt es im Zusammenhang mit den Kindern zu einem Absinken der Erwerbsquoten. Ähnlich wie im Westen fällt dieser Effekt umso deutlicher aus, je mehr Kinder erzogen werden. Der wesentliche Unterschied zum Ergebnis in den alten Bundesländern liegt aber darin, dass es relativ schnell wieder zu einem Anstieg kommt. Auch die Frauen mit einem und zwei Kindern erreichen dann wieder Werte von über $90 \%$. Gleiches gilt selbst für Frauen mit drei und mehr Kindern, die sich ebenfalls dieser Marke annähern. Anders als in Westdeutschland kommt es zu einem deutlich geringeren kinderbedingten Abfall der Werte. Die geringste Erwerbsquote von Frauen mit mehreren Kindern liegt bei nur $55 \%$.

\subsection{EINKOMMENSPOSITION}

Aber nicht nur bezogen auf die Erwerbsbeteiligung lässt sich der Einfluss von Kindererziehung nachzeichnen, sondern auch hinsichtlich der durchschnittlichen Einkommensposition (1 Entgeltpunkt=Durchschnittsverdiener). Besonders deutlich wird dies wiederum in den alten Bundesländern (Abbildung 3). Es sei jedoch vorab angemerkt, dass bei der Schilderung der nachfolgenden Befunde nicht abschließend geklärt werden kann, auf welche Einflussfaktoren dieser Einkommensunterschied zurückzuführen ist. Eine wichtige Erklärung liegt in der kind- bzw. familienbedingten Teilzeitbeschäftigung, die zu einer geringeren Entlohnung und damit zur Senkung des Durchschnitts führt.

Die Abbildungen 3 und 4 zeigen jeweils das durchschnittliche Einkommen aus sozialversicherungspflichtiger Beschäftigung. Zum Zeitpunkt der Untersuchung existierten die heute sozialversicherungsrelevanten sogenannten Mini- und Midijobs noch 
nicht, sodass diese nicht in die Werte mit eingehen.

Zunächst zur Einkommenssituation der Frauen in Westdeutschland (Abbildung 3): Zu Beginn der Berufsbiografie nimmt das durchschnittliche Erwerbseinkommen zuerst einen von der Anzahl der Kinder unabhängigen Verlauf. Ab dem 20. Lebensjahr beginnt eine Differenzierung zwischen den Frauen mit Kindern und den Frauen, die keine Kinder haben. Bei den kinderlosen Frauen gibt es eine ansteigende Entwicklung bis zum Alter von ca. 37 Jahren, wo im Mittel ein Entgeltpunkt, also ein im Sinne der Rentenversicherung durchschnittliches Einkommen, erzielt wird. Bei den Frauen mit Kindern zeigt sich kein weiterer Einkommensanstieg, bei Frauen mit zwei bzw. drei Kindern sinkt das durchschnittliche sozialversicherungspflichtige Einkommen sogar. Bei einem Kind wird langfristig ein durchschnittliches Einkommensniveau von 0,7 Entgeltpunkten erreicht. Bei zwei Kindern sinken die durchschnittlichen Entgeltpunkte, nachdem zunächst ebenfalls annähernd ein Wert von 0,7 erreicht wird, auf $0,55 \mathrm{ab}$ und verharren auch hier auf einem vergleichbaren Niveau. Frauen mit drei und mehr Kindern erreichen zuerst das vergleichsweise geringste durchschnittliche Entgelt von ca. 0,6 Entgeltpunkten, im nachfolgenden Verlauf sinkt dieser Wert auf 0,45 ab und steigt erst wieder ab dem 40. Lebensjahr leicht an.

Die Situation in Ostdeutschland ( $A b$ bildung 4) stellt sich anders dar. Auch dort zeigt sich ein kinderbedingter Effekt für die Jahrgänge 1936 bis 1955, dieser ist jedoch temporär. Die durch Kindererziehung verursachte Ausdifferenzierung der Durchschnitte beginnt zwischen dem 19. und 20. Lebensjahr. Bei den kinderlosen Frauen steigt das Einkommen im Mittel bis zum 25. Lebensjahr auf 0,8 Entgeltpunkte an und stabilisiert sich auf diesem Niveau bis zum ca. 40. Lebensjahr. Jetzt wiederum beginnt ein stetiges Absinken, das sich ab dem 50. Lebensjahr nochmals in einen Anstieg umkehrt. Bei Frauen mit Kindern bricht der Anstieg der durchschnittlichen Einkommen früher ab und es kommt zu einer mittelfristigen Stagnation der Werte. Gleiches gilt, je mehr Kinder zu erziehen sind. Bemerkenswert ist jedoch, dass es im folgenden Verlauf wieder zu einer Angleichung der Werte kommt, in dem die Frauen mit mehr Kindern aufholen; im 40. Lebensjahr sind die Werte für alle Gruppen dann nahezu gleich.

Abb. 4: Frauen in Ostdeutschland: Durchschnittliches Einkommen
aus sozialversicherungspflichtiger Beschäftigung (in Entgeltpunkten)
bezogen auf die Lebensjahre - Geburtsjahrgänge 1936-1955 mit eigener projizierter GRV-Rente -

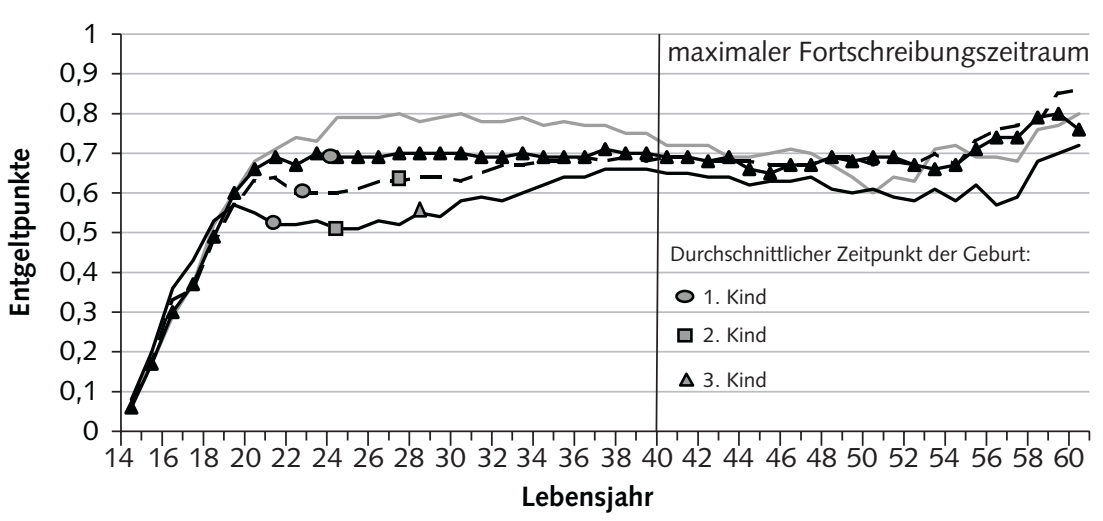

\begin{tabular}{l}
\hline keine Kinder $\longrightarrow$ ein Kind --- zwei Kinder - drei Kinder u.m. \\
Anm.: Maximaler Fortschreibungszeitraum beschreibt den maximalen Zeitraum, der durch die \\
Mikrosimulation mit statistisch ermittelten Werten gefüllt ist. Dieser ist am längsten beim Jahrgang \\
1955. \\
Quelle: Altersvorsorge in Deutschland 1996 (AVID 1996).
\end{tabular}

Das generelle Absinken der durchschnittlichen Entgelte mit einem anschließenden Anstieg in älteren Lebensjahren wird deutlich durch die besondere Situation in Ostdeutschland nach der Wiedervereinigung geprägt und ist im Wesentlichen auf die Arbeitsmarksituation zurückzuführen. Sowohl die empirischen Werte der älteren Kohorten als auch die Simulationsgrößen der jüngeren Jahrgänge für die späten Versicherungsjahre, die in die Nachwendezeit fallen, sind von hoher Arbeitslosigkeit bzw. arbeitsmarktbedingter Teilzeitarbeit geprägt. Die ansteigenden Durchschnitte in den Jahren vor dem 60. Lebensjahr sind dabei auch davon beeinflusst, dass besonders besser Qualifizierte (und damit in der Regel auch besser Entlohnte) deutlich eher in Beschäftigung verbleiben. Ein „Kindereffekt“ spielt hier keine direkte Rolle.

\section{Kindererziehung und Alterseinkommen}

Die durch Kindererziehung mehr oder weniger stark geprägten Erwerbsmuster haben Konsequenzen für die erwartete Höhe der Anwartschaften zur GRV-Rente und auf das gesamte Alterseinkommen. $\mathrm{Zu}$ nächst kann betrachtet werden, wie viele Entgeltpunkte sich in der Summe direkt aus sozialversicherungspflichtiger Beschäftigung ergeben und wie viele Entgeltpunkte insgesamt für die Berechnung der GRVRente maßgeblich sind. ${ }^{6}$ Die Differenz der beiden Werte spiegelt im Wesentlichen alle Regelungen wider, die besondere Lebenssituationen und -umstände anerkennen, welche direkt oder indirekt leistungssteigernd in die Bewertung der Rente einfließen (Bieber/Stegmann 2002).

In Tabelle 1 sind die errechneten Werte für verschiedene Personengruppen dargestellt. In den alten Bundesländern zeigt sich eine deutliche Differenzierung nach Anzahl der Kinder. Frauen der betrachteten Jahrgänge mit drei und mehr Kindern er-

6 Kernstück der Rentenberechnung ist die Ermittlung von Entgeltpunkten für Beitragszeiten und beitragsfreie Zeiten. Die Summe der Entgeltpunkte wird dann um Zuschläge erhöht oder um Abschläge vermindert, die sich aus besonderen rentenrechtlichen Sachverhalten ergeben (z. B. Zuschläge für beitragsgeminderte Zeiten oder Ausgleichszahlungen, Zu- oder Abschläge aus einem Versorgungsausgleich oder Ehegattensplitting). Nachdem aus dem gesamten Versicherungsleben Entgeltpunkte ermittelt werden, wird die Summe aller Entgeltpunkte in persönliche Entgeltpunkte umgerechnet. Dabei werden die Rentenabschläge bei einer vorzeitigen Inanspruchnahme gesteuert. Für einen persönlichen Entgeltpunkt erhält man die monatliche Rente, die ein Durchschnittsverdiener in einem Kalenderjahr erwirtschaftet hat. Im Jahre 2005 sind das $26,13 €$ in den alten und $22,97 €$ in den neuen Bundesländern. 
Tabelle 1: Frauen in Ost- und Westdeutschland: Durchschnittliche Entgeltpunktsummen aus sozialversicherungspflichtiger Erwerbstätigkeit und Summe der für die eigene GRV-Rente maßgeblichen Entgeltpunkte (EGPT) - Geburtsjahrgänge 1936-1955 mit eigener projizierter GRV-Rente -

\begin{tabular}{|c|c|c|}
\hline $\begin{array}{l}\text { AVID 1996: Geburtsjahrgänge } \\
\text { 1936-1955 } \\
\text { (nach RRG } 99 \text { ohne } \\
\text { Übergangsregelungen) }\end{array}$ & $\begin{array}{l}\text { Durchschnittl. Summe } \\
\text { der EGPT aus sozial- } \\
\text { versicherungspflichtiger } \\
\text { Erwerbstätigkeit }\end{array}$ & $\begin{array}{l}\text { Durchschnittl. Summe der } \\
\text { für die eigene GRV-Rente } \\
\text { maßgeblichen EGPT }\end{array}$ \\
\hline $\begin{array}{l}\text { Männer - alte Bundesländer } \\
\text { Männer - neue Bundesländer } \\
\text { Frauen - alte Bundesländer } \\
\text { Frauen - neue Bundesländer } \\
\text { Insgesamt }\end{array}$ & $\begin{array}{l}42.4 \\
37.3 \\
17.9 \\
26.3 \\
30.7\end{array}$ & $\begin{array}{l}45.7 \\
40.3 \\
22.8 \\
31.9 \\
34.8\end{array}$ \\
\hline \multicolumn{3}{|l|}{ Frauen nach Anzahl der Kinder } \\
\hline $\begin{array}{l}\text { Frauen - alte Bundesländer } \\
\text { keine Kinder } \\
1 \text { Kind } \\
2 \text { Kinder } \\
3 \text { Kinder und mehr }\end{array}$ & $\begin{array}{l}35.7 \\
20.6 \\
15.3 \\
10.7\end{array}$ & $\begin{array}{l}39.2 \\
24.8 \\
20.1 \\
17.2\end{array}$ \\
\hline $\begin{array}{l}\text { Frauen - neue Bundesländer } \\
\text { keine Kinder } \\
1 \text { Kind } \\
2 \text { Kinder } \\
3 \text { Kinder und mehr }\end{array}$ & $\begin{array}{l}30.6 \\
28.1 \\
26.7 \\
22.3\end{array}$ & $\begin{array}{l}34.1 \\
32.9 \\
32.1 \\
29.6\end{array}$ \\
\hline \multicolumn{3}{|l|}{$\begin{array}{l}\text { Frauen nach Voll- und Teilzeit } \\
\text { in der Erwerbsbiografie }\end{array}$} \\
\hline $\begin{array}{l}\text { Frauen - alte Bundesländer } \\
\text { Nur Vollzeit } \\
\text { Voll- und Teilzeit } \\
\text { Nur Teilzeit } \\
\text { Weder Voll- noch Teilzeit }\end{array}$ & $\begin{array}{r}7.2 \\
23.0 \\
10.2 \\
0.6\end{array}$ & $\begin{array}{r}10.6 \\
28.6 \\
14.6 \\
4.4\end{array}$ \\
\hline $\begin{array}{l}\text { Frauen - neue Bundesländer } \\
\text { Nur Vollzeit } \\
\text { Voll- und Teilzeit }\end{array}$ & $\begin{array}{l}18.1 \\
27.4\end{array}$ & $\begin{array}{l}24.6 \\
32.8\end{array}$ \\
\hline Quelle: AViD 1996; Berechnungen des Autors. & & WSI $\begin{array}{c}\text { Hans Böckle } \\
\text { Stiftung }\end{array}$ \\
\hline
\end{tabular}

Tabelle 2: Projizierte eigene Beteiligungen an Alterssicherungssystemen 1), projizierte Höhe der Anwartschaften auf Versichertenrenten der GRV und Nettoalterseinkommen in DM nach Zahl der Kinder2) (in Werten von 1996) - Geburtskohorten 1936-1955 mit eigener projizierter GRV-Rente -

\begin{tabular}{|c|c|c|c|c|c|c|c|c|}
\hline & \multicolumn{4}{|c|}{ Westdeutschland } & \multicolumn{4}{|c|}{ Ostdeutschland } \\
\hline & $\begin{array}{l}\text { Keine } \\
\text { Kinder }\end{array}$ & $\begin{array}{c}1 \\
\text { Kind }\end{array}$ & $\stackrel{2}{2}$ & $\begin{array}{l}3 \text { und } \\
\text { mehr } \\
\text { Kinder }\end{array}$ & $\begin{array}{l}\text { Keine } \\
\text { Kinder }\end{array}$ & $\begin{array}{c}1 \\
\text { Kind }\end{array}$ & $\stackrel{2}{\text { Kinder }}$ & $\begin{array}{l}3 \text { und } \\
\text { mehr } \\
\text { Kinder }\end{array}$ \\
\hline \multicolumn{9}{|l|}{ Alleinstehende Frauen } \\
\hline $\begin{array}{l}\text { Projizierte GRV-Anwartschaft } \\
\text { im 65. Lebensjahr }(\mathrm{DM})^{3)}\end{array}$ & 1.820 & 1.295 & 1.149 & 918 & 1.210 & 1.220 & 1.155 & 1.001 \\
\hline $\begin{array}{l}\text { Projiziertes persönliches } \\
\text { Netto-Alterseinkommen } \\
(\mathrm{DM})^{4)}\end{array}$ & 2.440 & 1.876 & 1.809 & 1.478 & 1.396 & 1.795 & 1.487 & 1.316 \\
\hline \multicolumn{9}{|l|}{ Verheiratete Frauen } \\
\hline $\begin{array}{l}\text { Projizierte GRV-Anwartschaft } \\
\text { im 65. Lebensjahr }(D M)^{3)}\end{array}$ & 1.503 & 971 & 788 & 669 & 1.160 & 1.124 & 1.119 & 1.041 \\
\hline $\begin{array}{l}\text { Projiziertes persönliches Netto- } \\
\text { Alterseinkommen }(\mathrm{DM})^{4)}\end{array}$ & 1.806 & 1.156 & 968 & 807 & 1.310 & 1.280 & 1.310 & 1.162 \\
\hline \multicolumn{9}{|l|}{ Ehepaare ${ }^{5)}$} \\
\hline $\begin{array}{l}\text { Summe der projizierten } \\
\text { GRV-Anwartschaft beider } \\
\text { Ehepartner }(\mathrm{DM})^{4)}\end{array}$ & 3.282 & 2.955 & 2.763 & 2.490 & 2.345 & 2.560 & 2.555 & 2.396 \\
\hline $\begin{array}{l}\text { Summe des projizierten Netto- } \\
\text { Alterseinkommens beider } \\
\text { Ehepartner (DM)5) }\end{array}$ & 4.332 & 3.935 & 3.800 & 3.261 & 2.776 & 2.973 & 3.029 & 2.822 \\
\hline \multicolumn{9}{|c|}{$\begin{array}{l}\text { 1) Einbezogene Systeme: GRV, BAV, ZÖD, BV, AdL, BSV und PV (LV/PRV) } \\
\text { 2) Familienstand gemäß der Situation im Jahr } 1996 \\
\text { 3) Zahlbetrag vor Einkommenteuer und nach Abzug des Eigenanteils zur Kranken- und Pflegeversicherung der Rentner } \\
\text { 4) Zahlbetrag nach Abzug der Einkommensteuer und des Eigenanteils zur Kranken- und Pflegeversicherung } \\
\text { 5) Ehemann der Geburtskohorten 1936-1955 mir projizierter Anwartschaft auf Versichertenrente der GRV }\end{array}$} \\
\hline & & & & & & WS & S1 Hans & $\begin{array}{l}\text { Böckler } \\
\text { ung }\end{array}$ \\
\hline
\end{tabular}

reichen im Durchschnitt 10,7 Entgeltpunkte aus sozialversicherungspflichtiger Beschäftigung, der Wert für die kinderlosen Frauen liegt dagegen bei 35,7 Entgeltpunkten. Nimmt man alle Entgeltpunkte, die für die Rentenberechnung maßgeblich sind, dann kommen die Frauen mit drei Kindern auf 17,2 Entgeltpunkte, während die kinderlosen Frauen im Mittel auf einen Wert von 39,2 kommen. Die Kindererziehung und die damit verbundene geringe Beteiligung am Erwerbsleben sowie die daran gekoppelte spezifische Situation hinsichtlich der Entlohnung können durch zusätzliche Leistungen der GRV nur teilweise ausgeglichen werden.

In den neuen Bundesländern verhält es sich für die betrachteten Geburtsjahrgänge anders. Der kinderbedingte Unterschied in den durchschnittlichen Entgeltpunktsummen aus sozialversicherungspflichtiger Beschäftigung fällt deutlich geringer aus; er beträgt zwischen den kinderreichen und den kinderlosen Frauen acht Punkte und nur 4,5 Entgeltpunkte bezogen auf die letztlich für die Rente maßgeblichen Anwartschaften. Im Vergleich zum Westen liegt dies am unterschiedlichen Muster der Erwerbsbeteiligung, aber auch an dem vergleichsweise geringeren Unterschied hinsichtlich der Höhe der Beiträge und damit auch der Entlohnung.

In Tabelle 1 wird auch deutlich, dass Frauen mit einer Mischbiografie aus Vollund Teilzeittätigkeit im Durchschnitt die höchsten GRV-Anwartschaften erzielen (Kortmann/Schatz 1999).Zurückzuführen ist dieses Ergebnis auf die unterschiedliche Dauer der sozialversicherungspflichtigen Tätigkeit. Betrachtet man den Zusammenhang zwischen der Dauer der Phasen mit Teilzeittätigkeit und den je durchschnittlich durchlebten Jahren mit Vollzeittätigkeit, wird offensichtlich, dass die Teilzeitbeschäftigung ein wichtiges Bindeglied zur Arbeitswelt darstellt (ausführlich: Bieber/ Stegmann 2000).

Kritisch zu hinterfragen ist danach die These, dass die niedrigen Entgelte, die während der Teilzeitphasen erworben werden, auch zu niedrigen Renten führten und dass teilzeitarbeitende Frauen ,am schlechtesten abschneiden" (MfKJFF 1997). Sicherlich ist es richtig, dass eine fiktive Frauen-Biografie mit 40 Jahren in Teilzeittätigkeit bei „typischem Frauenlohn“ zu einer im Vergleich zur Standardrente niedrigen Anwartschaft führt (ebd.). Doch diese eher typologisch denn empirisch begründete 
These verdeckt den Zusammenhang, nach dem Phasen der Teilzeittätigkeit als Scharniere zwischen Vollzeitarbeit- und Familienphasen dienen oder die Erwerbstätigkeit während längerer Phasen der Haushaltsführung ermöglichen. Durch diese Wirkung erhöhen sich die Anwartschaften teilzeitarbeitender Frauen bei Renteneintritt letztlich in wesentlichem Umfang. Die Alternative zur Teilzeitbeschäftigung ist unter den gegebenen Verhältnissen am Arbeitsmarkt, in den Familien und in der Kinderbetreuung nicht Vollzeiterwerbstätigkeit, sondern Erwerbslosigkeit.

Die Auswirkungen der Kindererziehung spiegeln sich auch in den Befunden zum gesamten Nettoalterseinkommen wider (Tabelle 2), bei denen neben der gesetzlichen Rente auch Betriebsrenten und private Vorsorge berücksichtigt werden. Dabei muss hervorgehoben werden, dass es sich bei den vorgestellten Beträgen um Werte des Jahres 1996 handelt, dem Bezugsjahr der AVID 1996. In den alten Ländern besteht ein inverser Zusammenhang zwischen der Zahl der Kinder und der Anzahl der Beteiligungen an Alterssicherungssystemen sowie der Höhe der GRVAnwartschaften. Sowohl bei alleinstehenden Frauen als auch bei verheirateten Frauen sinkt das projizierte Renteneinkommen und das persönliche Nettoalterseinkommen in Abhängigkeit von der Kinderzahl. Dies zeigt auch Auswirkungen auf die Situation im Ehepaarbezug. Auf Ebene der Netto-Alterseinkommen verstärken sich diese Unterschiede, da kinderlose Frauen bzw. Ehepaare eher in der Lage waren und sind, (größere) finanzielle Mittel für eine zusätzliche Altersvorsorge aufzubringen. So erreichen Ehepaare ohne Kinder ein deutlich höheres Alterseinkommen als Kinderreiche. Für die neuen Bundesländer lässt sich ein solch eindeutiger $\mathrm{Zu}$ sammenhang nicht nachvollziehen, eher besteht eine gegenteilige Tendenz. Auch auf der individuellen Ebene erreichen Frauen mit Kindern kein geringeres persönliches Netto-Alterseinkommen, im Gegenteil erzielen Frauen mit Kindern sogar höhere Werte.

\section{0 \\ Fazit}

Zusammenfassend ist festzustellen, dass Kinder bei den meisten Müttern der untersuchten Geburtskohorten zu einem zumindest zeitweiligen Ausstieg aus dem Erwerbsleben führen. Je mehr Kinder eine Frau hat, desto eher ist dies der Fall und umso länger ist die Dauer der Kinderpause bzw. umso größer ist die Wahrscheinlichkeit, dass es zu einem dauerhaften Ausstieg kommt. Diese Befunde zeigen sich vor allem in den alten Bundesländern, in Ostdeutschland treten sie im Untersuchungszeitraum nur deutlich abgemildert hervor. Dort gelang auch kinderreichen Frauen zu einem hohen Anteil ein beruflicher Wiedereinstieg.

Als weiteres Ergebnis lässt sich festhalten, dass im Zusammenhang mit der Kindererziehung das durchschnittlich erzielte Einkommen der berufstätigen Mütter hinter dem der kinderlosen Frauen zurückbleibt, und zwar umso stärker, je mehr Kinder die Frauen haben. In Westdeutschland wird dieser durchschnittliche Abstand langfristig nicht wieder aufgeholt. In Ostdeutschland ist dieser Einkommensunterschied für die betrachteten Jahrgänge nur auf den Zeitraum der Kindererziehung bezogen und es kommt relativ schnell wieder zu einer Angleichung der Verhältnisse. Die geringere relative Einkommensposition von Müttern ist zum Teil auf die Teilzeiterwerbstätigkeit zurückzuführen. Diese hat aber auch eine wichtige Bedeutung, da sie häufig eine Brückenfunktion zur Vollzeittätigkeit ist. In der Summe betrachtet erreichen die Frauen mit einer Mischbiografie aus Voll- und Teilzeiterwerbstätigkeit die höchsten Rentenanwartschaften.

Die kinderspezifischen Erwerbsmuster schlagen sich in den alten Bundesländern deutlich in den Anwartschaften auf die GRV-Rente nieder und können auch durch besondere Leistungen der gesetzlichen Rentenversicherung, insbesondere kinderbezogene Leistungen, nicht ausgeglichen werden. Auch bezogen auf das Gesamtalterseinkommen erreichen Frauen mit Kindern bzw. Ehepaare mit Kindern ein geringeres Niveau als Kinderlose. Dies wird umso deutlicher, je mehr Kinder vorhanden sind. In den neuen Bundesländern ergibt sich kein kinderbezogener Nachteil in der GRV-Rente und im Alterseinkommen insgesamt. Es kommt im Gegenteil zu höheren Beträgen bei Frauen mit einem oder zwei Kindern im Vergleich zu Kinderlosen. $\mathrm{Zu}$ bemerken ist dabei, dass bei den betrachteten Jahrgängen die Kindererziehungszeiten in der Regel ein Jahr betragen, weil die Kinder vor 1992 geboren wurden. Die dreijährige Kindererziehungszeit für Geburten ab 1992 und die Höherbewertung von Zeiten der Kindererziehung bei Erwerbstätigkeit nach dem AvmEG ${ }^{7}$ schlagen hier nicht zu Buche. Sie betreffen zumeist jüngere Jahrgänge. Modellrechnungen zeigen aber auch hier, dass sie zwar zu Verbesserungen führen, jedoch die kinderbezogenen Beitragsausfälle nicht kompensieren.

Die betriebliche und private Vorsorge spielt bei den betrachteten Geburtsjahrgängen in Westdeutschland eine deutlich größere Rolle als in Ostdeutschland. Dabei zeigt sich, dass es hier ebenfalls zu deutlichen Einflüssen der Kindererziehung kommt. Im Ehepaarkontext ergibt sich in der Folge ein deutlich niedrigeres durchschnittlich projiziertes Gesamtalterseinkommen von kinderreichen Ehepaaren im Vergleich zu kinderlosen. Auch hier wird die Notwendigkeit deutlich, besonders bei Frauen mit mehreren Kindern die zusätzliche Altersvorsorge zu unterstützen.

\footnotetext{
7 AvmEG: Gesetz zur Ergänzung des Gesetzes zur Reform der gesetzlichen Rentenversicherung und zur Förderung eines kapitalgedeckten Altersvorsorgevermögens (Altersvermögensergänzungsgesetz).
} 
Beckmann, P. (2002): Zwischen Wunsch und Wirklichkeit. Empirische Ergebnisse zur Teilzeit. Bundesarbeitsblatt 11, S. 13-17

Bieber, U./Stegmann, M. (2000): Sozialversicherungspflichtige Teilzeitbeschäftigung in den Erwerbsbiographien der zukünftigen Rentnerinnen. Eine Kohortenbetrachtung auf Basis der Untersuchung Altersvorsorge in Deutschland 1996 (AVID 1996), Deutsche Rentenversicherung 6, S. 364 383

Bieber, U./Stegmann, M. (2002): Maßnahmen des sozialen Ausgleichs innerhalb der Gesetzlichen Rentenversicherung, Relevante Anwartschaftsbestandteile bei den Geburtsjahrgängen 1936 bis 1955, Deutsche Rentenversicherung 11, S.612-641

Hauschild, C. (2002): Die empirische Typisierung von Versichertenbiografien, Deutsche Rentenversicherung 9-10, S. 539-590

Klammer, U./Tillmann, K. (2001): Vielfalt als Normalität? - Querschnittsergebnisse zur Ausdifferenzierung der Arbeitsverhältnisse in Deutschland und NRW, in: WSI in der Hans Böckler-Stiftung (Hrsg.): Flexicurity: Soziale Sicherung und Flexibilisierung der Arbeits- und Lebensverhältnisse, Düsseldorf/Ministerium für Arbeit und Soziales, Qualifikation und Technologie des Landes Nordrhein-Westfalen, S. 45-82

Klöpfer, K. (1954): Reichsversicherungs-Ordnung, 40. Auflage, Stuttgart Kortmann, K./Schatz, C. (1999): Altersvorsorge in Deutschland 1996 (AVID 1996), Lebensverläufe und künftige Einkommen im Alter, in: Verband Deutscher Rentenversicherungsträger und Bundesministerium für Arbeit und Sozialordnung (Hrsg.): DRV-Schriften 19 und BMA-Forschungsbericht 277, München

Kortmann, K./KneißI, G./Schatz, C. (2000): Altersvorsorge in Deutschland 1996 (AVID 1996) - Phase 1 und 2 Datenerhebung und -aufbereitung, in: Verband Deutscher Rentenversicherungsträger und Bundesministerium für Arbeit und Sozialordnung (Hrsg.): Methodenbericht von Infratest

Burke Sozialforschung, München

Krauthausen, H. (2002): Auf dem Weg zu einer eigenständigen Alters- sicherung von Frauen, Bundesarbeitsblatt 6, S. 27-32

Ministerium für Kultur, Jugend, Familie und Frauen (MfKJFF) RheinlandPfalz (Hrsg.) (1997): Die systematische Benachteiligung von Frauen im Rentenrecht, Mainz

Mika, T./Bieber, U. (2006): Verdeckte Armut der älteren Bevölkerung, Ausmaß von Niedrigeinkommen und Gründe der Nichtinanspruchnahme von Sozialhilfe unter Senioren. Deutsche Rentenversicherung 4-5,

S. 248-279

Pabel, F. (1998): Familie und Frauen in der gesetzlichen Rentenversicherung, Mitteilungen der LVA Baden-Württemberg 7-8, S. 169-174

Polster, A., (1998): Kindererziehungszeiten besser bewertet, Deutsche Rentenversicherung 1-2, S. 71-79

Prinz, K., (1997): Versicherungsverläufe von Frauen und Männern der Geburtsjahrgänge 1931 bis 1960, Deutsche Rentenversicherung 3-4, S. $220-241$

Rahn, M./Becker, S. (1997): Reform der sozialen Sicherung der Frau Bestandsaufnahme und Perspektiven aus deutscher und internationaler Sicht, Deutsche Rentenversicherung 11-12, S. 662-689

Ruland, F. (2001): Familie und Rentenversicherung, Mitteilungen der LVA Mittel- und Oberfranken 12, S. 699-705

Stegmann, M. (2001): Die Höherbewertung von Pflichtbeiträgen während Zeiten der Kindererziehung, Abschätzung der Auswirkung auf Basis der AVID 1996, in: Deutsche Rentenversicherung 12, S. 753-770

Stegmann, M. (2003): Empirische Fakten und Trends zur soziodemografischen Situation und zur Alterssicherung von Frauen, Deutsche Rentenversicherung 3-4, S. 161-188

Stegmann, M. (2004): Eskalation an der Küchenzeile? Einstellungen zur Erwerbstätigkeit der Mütter während der (Klein)kindphase, in: Deutsche Rentenversicherung 4, S. 547-560

Verband Deutscher Rentenversicherungsträger (VDR) (Hrsg.) (1998):

VDR Statistik Aktiv Versicherte 1995/1996, Frankfurt am Main 